\title{
Using Syndromic Surveillance to Enhance Arboviral Surveillance in Arizona
}

\author{
Jessica R. White ${ }^{\star 1}$, Sara Imholte ${ }^{2}$ and Krystal Collier ${ }^{2}$ \\ 'Epidemiology, Maricopa County Department of Public Health, Phoenix, AZ, USA; ${ }^{2}$ Arizona Department of Health Services, Phoenix, \\ AZ, USA
}

\section{Objective}

To develop a protocol for enhancing traditional arboviral surveillance with syndromic surveillance and to evaluate the protocol for accuracy, effectiveness, and timeliness.

\section{Introduction}

Arizona is facing multiple public health threats from arboviral diseases. State and local public health departments are monitoring two mosquito-borne outbreaks within its borders and two in adjacent territories. To prevent transmission, viremic patients must be identified in a timely manner and encouraged to avoid additional mosquito exposure and vector control actions must be implemented. Using traditional surveillance, however, health departments may not be alerted until the laboratory confirms and reports a positive result, which may take up to 14 days after specimen collection. The Arizona Department of Health Services (ADHS) partnered with local public health jurisdictions to enhance traditional arboviral surveillance by incorporating syndromic surveillance.

\section{Methods}

In collaboration, ADHS and county epidemiologists developed a protocol for identifying and responding to possible arboviral cases using chief complaint and diagnosis data from the National Syndromic Surveillance Platform. Arizona's Arboviral Syndromic Surveillance Protocol outlines steps for both state and county public health staff. In phase 1, ADHS uses phpMyAdmin two times per week to extract emergency department and inpatient visit data. The query retrieves records that include variant spellings for chikungunya, dengue, West Nile virus, or St. Louis encephalitis. These records are posted to county-specific spreadsheets on a secure server. In phase 2, counties use a decision tree to determine whether patient visits warrant further investigation (Figure). For visits of interest, staff note whether the patient was previously reported via traditional surveillance and whether the medical record provides additional information to change the level of suspicion. Counties decide whether to contact the patient to gather more information, provide education, or alert their vector control department.

\section{Results}

This abstract was submitted 1.5 weeks after Arizona officially began using syndromic surveillance for enhanced arboviral surveillance (on 8/24/15). After 3 data extractions by ADHS, records for 12 visits from 11 unique patients were retrieved by the arboviral query. The patient records, from 2 Arizona counties, mentioned West Nile virus (10) and St. Louis encephalitis (1). Eight of the 11 patients were previously identified via traditional surveillance. Medical records from the remaining 3 patients were reviewed. Arboviral diseases were not confirmed by the medical records of these 3 patients.

\section{Conclusions}

In collaboration with county health departments, ADHS developed a protocol for identifying and responding to possible arboviral cases using syndromic surveillance. Initially, the query, which searches for records that specifically mention an arboviral disease, retrieved 11 unique patients from 2 counties. Dividing responsibilities between ADHS and county staff has been helpful for managing time and resources for this enhanced surveillance effort. ADHS plans to continue using syndromic surveillance for arboviral surveillance through January 2016. As data are collected, state and county epidemiologists will monitor the protocol's effectiveness and timeliness and implement modifications if necessary. In January, a full evaluation will assess the query's ability to identify true positive cases, the speed at which possible cases are identified compared to traditional surveillance, and the time dedicated by state and county staff for enhanced surveillance. As the evaluation continues, the authors will continue to share the findings.

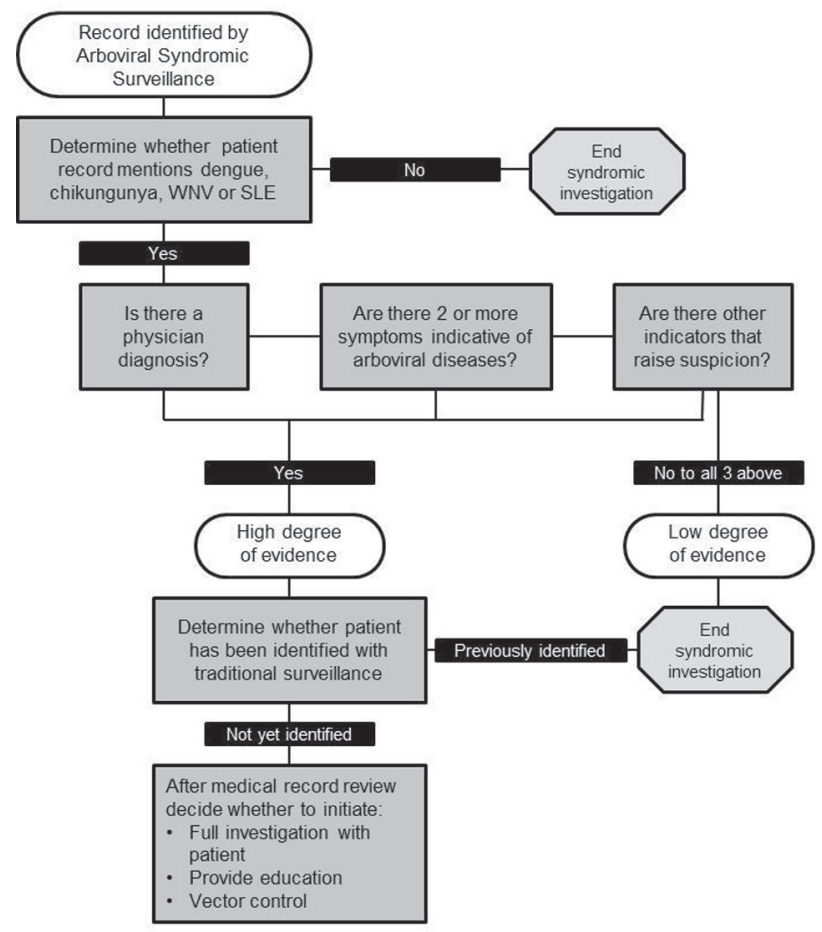

Figure. Decision tree for triaging arboviral records identified through syndromic surveillance

\section{Keywords}

arboviral; syndromic; use case; collaborative

\section{Acknowledgments}

The authors thank the arboviral and syndromic teams at both ADHS and Maricopa County Department of Public Health for their contributions.

\section{*Jessica R. White}

E-mail: JessicaWhite@mail.maricopa.gov 